\title{
Note on Transliteration and Dates
}

In keeping with standard scholarly practice, we have used the Library of Congress transliteration system to render Russian names and words, except where there is a well-established English equivalent.

Dates are indicated in the Old (Julian) Style except where otherwise noted. 
